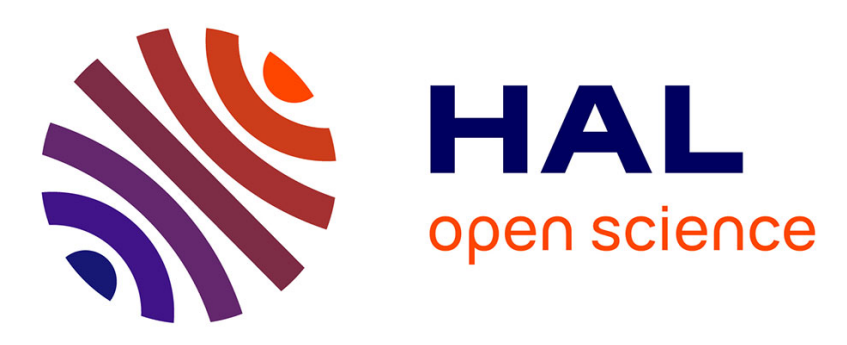

\title{
Three-dimensional phononic crystal with ultra-wide bandgap at megahertz frequencies
}

Julio Andrés Iglesias Martinez, Johnny Moughames, Gwenn Ulliac, Muamer Kadic, Vincent Laude

\section{- To cite this version:}

Julio Andrés Iglesias Martinez, Johnny Moughames, Gwenn Ulliac, Muamer Kadic, Vincent Laude. Three-dimensional phononic crystal with ultra-wide bandgap at megahertz frequencies. Applied Physics Letters, 2021, 118 (6), pp.063507. 10.1063/5.0033615 . hal-03186585

\section{HAL Id: hal-03186585 https://hal.science/hal-03186585}

Submitted on 31 Mar 2021

HAL is a multi-disciplinary open access archive for the deposit and dissemination of scientific research documents, whether they are published or not. The documents may come from teaching and research institutions in France or abroad, or from public or private research centers.
L'archive ouverte pluridisciplinaire HAL, est destinée au dépôt et à la diffusion de documents scientifiques de niveau recherche, publiés ou non, émanant des établissements d'enseignement et de recherche français ou étrangers, des laboratoires publics ou privés. 


\section{Three-Dimensional Phononic Crystal with Ultra-Wide Bandgap at Megahertz Frequencies}

Julio Andres Iglesias Martinez, ${ }^{\text {a) }}$ Johnny Moughames, Gwenn Ulliac, Muamer Kadic, and Vincent Laude Institut FEMTO-ST, CNRS UMR 6174, Univ. Bourgogne Franche-Comté, Besançon, France

Phononic crystals have attracted wide attention in diverse scientific communities due to their ability to efficiently reflect, focus, and guide acoustic and elastic waves. Their use in ultrasonic applications such as medical imaging, however, remains elusive since three-dimensional phononic crystals with lattice constants in the range of hundreds of micrometers are required to operate with $\mathrm{MHz}$ ultrasound, smaller than what most additive manufacturing solutions can provide. This work presents a three-dimensional phononic crystal with a cubic symmetry having an experimental ultra-wide complete bandgap extending from $0.6 \mathrm{MHz}$ to $7.5 \mathrm{MHz}$. Crystal specimens are manufactured using two-photon lithography at the microscale, with a lattice constant of $300 \mu \mathrm{m}$, and are characterized using heterodyne interferometry.

Phononic crystals are artificial periodic structures designed to mimic the acoustical properties of lattice phonons at much larger scales ${ }^{1,2}$. Their properties extend beyond those of typical lattice phonons and, in this sense, they form a subclass of metamaterials ${ }^{3-11}$. For the last 30 years, they have found applications as reflectors and filters ${ }^{2}$, but they have also been helpful in topological physics ${ }^{12}$ or for polarization control ${ }^{13-16}$. Their main feature is the fundamental Bragg bandgap that occurs when the effective wavelength roughly equals twice the lattice constant of the crystal. In addition, many researchers have explored local resonances to achieve bandgaps at low frequencies, for audible sound or seismic applications ${ }^{17,18}$.

Ultrasonics as a discipline has paved the way for widely used medical imaging techniques such as echography. Ultrasonic frequencies result from a compromise between axial resolution and material losses that are inherent in human tissues. They typically extend from 1 to $15 \mathrm{MHz}^{19,20}$. Three-dimensional phononic crystals operating at ultrasonic frequencies, however, remain elusive. Solid 3D phononic crystals can, indeed, be arranged by hand by embedding an array of millimeter-size steel balls in epoxy ${ }^{21}$ or paraffin ${ }^{22}$. The resulting phononic bandgap then appears around $500 \mathrm{kHz}$, see Table I, but the unit cell cannot be optimized. Recently, additive manufacturing has been shown to enable the realization of complex unit cell designs and thus the optimization of the bandgap width ${ }^{23-26}$. Because of the limited resolution of 3D printers, available frequencies remain well below 1 $\mathrm{MHz}$.

Recently, two-photon lithography (TPL), a 3D direct laser writing technique inside polymers, has enabled researchers to manufacture objects with an overall size of few millimeters and smallest features as small as $100 \mathrm{~nm}$. TPL even enables the fabrication of overhanging parts that would appear to violate the law of gravity if one forgets about the viscosity of monomers and the extreme writing speeds ${ }^{13}$ that leave almost no time for the parts

\footnotetext{
a) Electronic mail: julio.iglesias@femto-st.fr
}

Table I: 3D phononic crystals. pc: perovskite-cubic; fcc: face-centered cubic; hcp: hexagonal compact; sc: simple cubic; bcc: body-centered cubic; SLS:selective laser sintering; SLA: stereolithography apparatus; SBEM:

Selective Electron Beam Melting.

\begin{tabular}{cccc}
\hline Symmetry & Fabrication & $\omega_{g}$ & $\Delta \omega / \omega_{g}$ \\
\hline \hline pc [this work] & 3D printing (TPL) & $4 \mathrm{MHz}$ & $170 \%$ \\
$\mathrm{fcc}^{21}$ & steel balls and epoxy & $500 \mathrm{kHz}$ & $60 \%$ \\
$\mathrm{hcp}^{22}$ & steel balls and paraffin & $635 \mathrm{kHz}$ & $72 \%$ \\
$\mathrm{pc}^{23}$ & 3D printing (SLS) & $11.36 \mathrm{kHz}$ & $132 \%$ \\
$\mathrm{sc}^{25}$ & 3D printing (SLA) & $55 \mathrm{kHz}$ & $166 \%$ \\
$\mathrm{bcc}^{26}$ & 3D printing (SLS) & $11.3 \mathrm{kHz}$ & $48 \%$ \\
$\mathrm{sc}^{24}$ & 3D printing (SBEM) & $90 \mathrm{kHz}$ & $22 \%$ \\
\hline
\end{tabular}

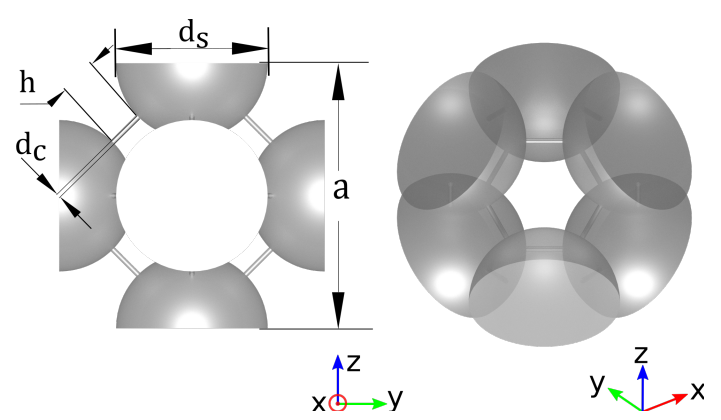

Figure 1: Schematic view of the unit cell of the perovskite cubic three-dimensional phononic crystal. Designed dimensions are $a=300 \mu \mathrm{m}, h=40.8 \mu \mathrm{m}$,

$$
d_{s}=171.3 \mu \mathrm{m}, \text { and } d_{c}=6 \mu \mathrm{m} \text {. }
$$

to fall down before getting fully connected. This flexibility efficiently relieves fabrication and geometrical constraints. In this letter, we use the extraordinary flexibility of $3 \mathrm{D}$ direct laser writing ${ }^{27}$ to fabricate a phononic crystal that presents a wide complete bandgap at ultrasonic frequencies. The fabricated polymer structure is coated with a thin metal layer, to improve the reflectivity of the sample, and experimentally characterized using a scanning laser vibrometer.

Figure 1 depicts the unit cell of the phononic crystal 

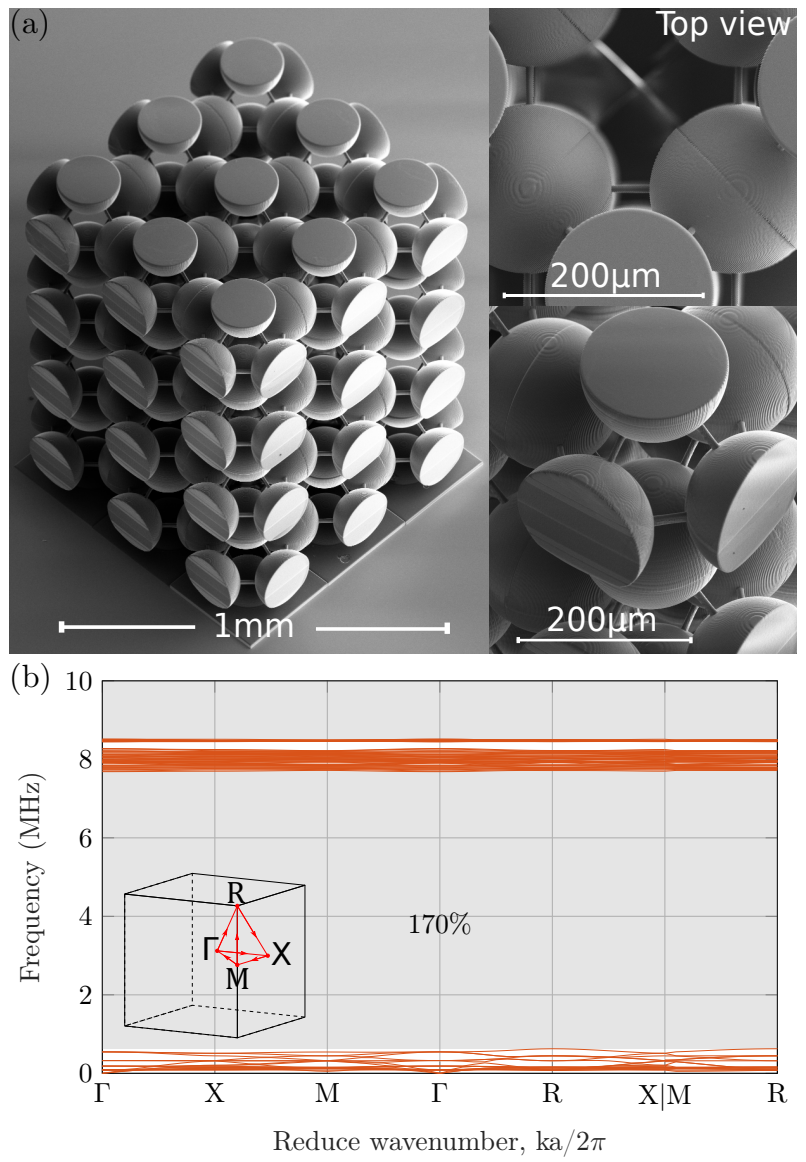

Figure 2: (a) SEM view of the phononic crystal sample fabricated by TPL. The phononic crystal is coated with a thin chromium coating to enhance vibration metrology. The top plate is here removed to show the internals of the crystal but was present during vibration experiments. (b) Phononic band structure obtained with the finite element method, with complete bandgaps outlined in gray.

that we consider. The space group is chosen to conform to symmetries of the perovskite structure, in the class of cubic crystals. Previous studies have indicated that very wide phononic bandgaps can be achieved based on this structure, benefiting from both Bragg scattering and local resonance ${ }^{23,25}$. Each cubic unit cell contains three balls connected by thin bars. We set the diameter of the bars to a small value ensuring mechanical stability during TPL, $d_{c}=6 \mu \mathrm{m}$. Once the lattice constant is fixed as $a=300 \mu \mathrm{m}$, only one free geometrical parameter remains. Optimization of the bandgap width then yields $h=40.8 \mu \mathrm{m}$ and $d_{s}=171.3 \mu \mathrm{m}$. The filling fraction is $39 \%$. The geometrical dimensions are chosen to respect fabrication constraints.

Figure 2(a) shows a scanning electron micrograph (SEM) view of the fabricated phononic crystal. Twophoton polymerization (Nanoscribe, model GT) was performed with a negative tone photoresist "Ip-S" ${ }^{13}$ dropped
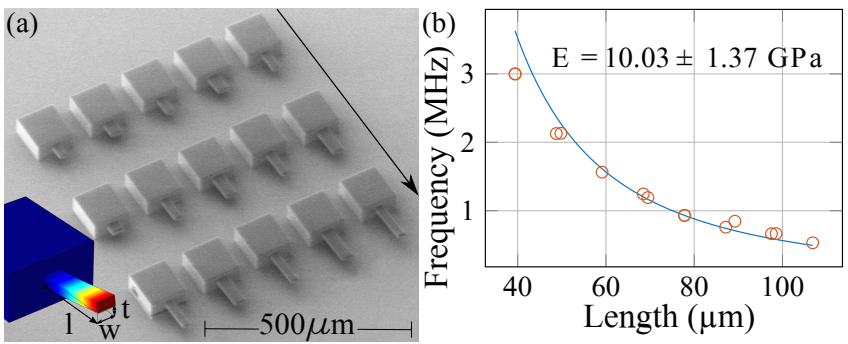

Figure 3: (a) SEM view of fabricated cantilevers with various length. (b) Measured resonance frequency $f_{b}$ of the fundamental bending mode of the cantilever versus length, $l$. Formula $2 \pi f_{b}=(1.875)^{2} \sqrt{E / 12 \rho}\left(t / l^{2}\right)$, valid under Euler-Bernoulli theory for beams with a rectangular cross-section, is used for the fit.

on a PZT-5A piezoelectric patch of $5 \times 7 \times 0.5 \mathrm{~mm}$. The femtosecond laser beam, centered at $780 \mathrm{~nm}$, was focused by a $25 \times(0.8 \mathrm{NA})$ objective lens. After the writing, the sample was immersed in a PGMEA (1-methoxy2-propanol acetate) solution for 20 minutes to remove the monomer photoresist. The scanning mode (at constant speed $100 \mathrm{~mm} / \mathrm{s}$ ) with a goniometric mirror was used. Post-fabrication measurements yield $a=299.6 \mu \mathrm{m}$, $h=37.1 \mu \mathrm{m}, d_{s}=170.8 \mu \mathrm{m}$, and $d_{c}=6.6 \mu \mathrm{m}$ for the crystal sample.

The phononic properties shown in Figure 2(b) are evaluated numerically with the finite element method $^{2}$. The phononic band structure is obtained by solving the elastodynamic equation

$$
-\rho \omega^{2} \mathbf{u}=\frac{E}{2(\nu+1)} \nabla(\nabla \cdot \mathbf{u})+\frac{E}{\nu+1} \nabla^{2} \mathbf{u}
$$

where $\rho$ is the mass density, under Bloch periodic boundary conditions. Furthermore, transmissions are calculated using a frequency sweep under a time-harmonic excitation of the bottom surface of the crystal. In order to evaluate the Young's modulus of our samples, we have fabricated cantilevers with various length $l$ using the same writing parameters as for the crystals. A SEM image of the fabricated cantilevers is shown in Figure 3(a), with thickness $t=12 \mu \mathrm{m}$ and width $w=40$ or $20 \mu \mathrm{m}$. The vibration frequency of the fundamental bending mode of the cantilever is measured and compared with its theoretical value given by Euler-Bernoulli beam theory ${ }^{28}$ in Figure 3(b). A fit to the model leads to Young's modulus $E \approx 10 \mathrm{GPa}$. The polymer is here considered isotropic. Poisson's ratio $\nu=0.43$ and mass density $\rho=1200 \mathrm{~kg} / \mathrm{m}^{3}$ are determined experimentally for the laser power used during writing.

The crystal, designed assuming a lossless polymeric material, has a theoretical complete bandgap extending from 0.6 to $7.7 \mathrm{MHz}$, as Figure 2(b) shows. The anisotropic universal factor ${ }^{29}$ equals 0.52 , which is much closer to the isotropic value of 0 than for the $\mathrm{BCC}$ symmetry $^{30}$ and the SC symmetry ${ }^{25}$, for which it can be calculated to be around 31 and 28 , respectively. In the 

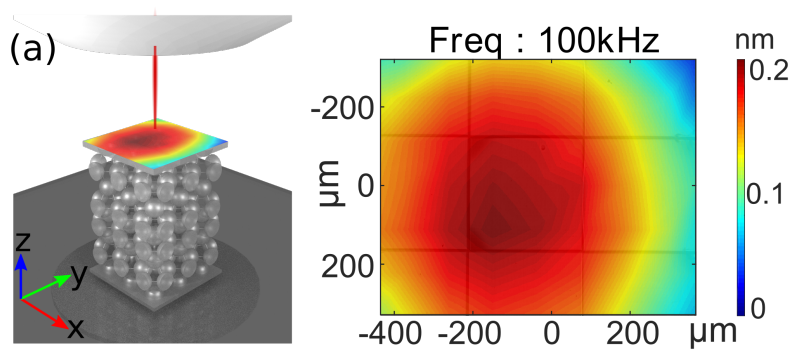

(b)

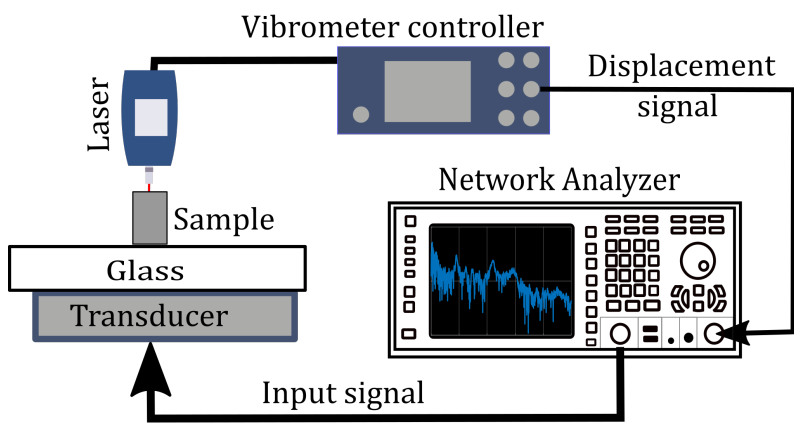

Figure 4: (a) Principle of vibration metrology. The sample is placed under the scanning laser beam and its reflection is recorded in time. An example of a scan of

the out-of-plane displacement field at $100 \mathrm{kHz}$ is overlaid on the top surface. (b) Schematic of the experimental setup.

experimental validation, we test only direction [100], or $\Gamma X$. Though other symmetry directions should in theory be included as well, especially $\Gamma R$, band foldings along the $\Gamma \mathrm{X}$ direction imply that the gap width is almost the same in all principal directions. Furthermore, in the experiments we used a source of longitudinal elastic waves and we detected out-of-plane vibrations only. It could then be questioned if shear waves may propagate through the structure when longitudinal waves cannot. In the case of 3D phononic crystals, however, there is generally no decoupling of shear and longitudinal waves and we thus do not expect the presence of shear vibrations in the absence of out-of-plane vibrations.

Figure 4 illustrates the experimental set-up. Out-ofplane displacements were measured with a laser Doppler vibrometer (Polytec, model MSA 500). For the source of elastic waves, we used a $0.25 \mathrm{~mm}$ thick and $6 \mathrm{~mm}$ wide PZT patch (PI Ceramic PIC255) in longitudinal mode. A network analyzer (Rhode \& Schwartz, model ZV) measured the transmittance parameter $S_{12}$, with port 1 connected to the transducer and port 2 receiving the output displacement signal from the vibrometer. The input power signal was $20 \mathrm{dBm}$. The bandwidth of the measurements was set to $100 \mathrm{~Hz}$, with an average factor of 10 to reduce measurement noise. An example of a singlefrequency scan of the top surface is shown in Fig. 4(a). Measurements were performed in several positions at the top surface and were then averaged to improve the signal to noise ratio.
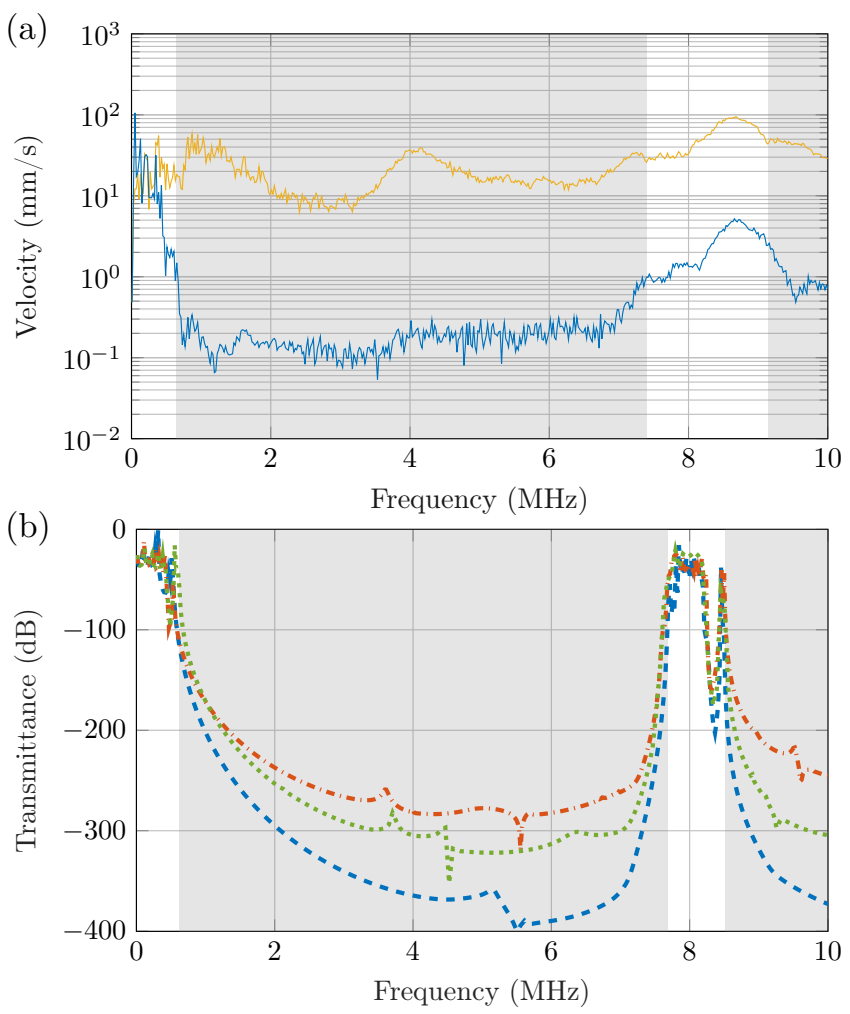

Figure 5: (a) Experimental out-of-plane displacements measured by laser Doppler vibrometry. Incident vibrations (orange line) are measured on top of the glass substrate covering the PZT transducer. Vibrations for the phononic crystal sample (blue line) are measured at the top surface of the TPL samples. (b) Numerical transmission computed by finite element analysis for directions $\Gamma X$ (blue dashed line), ГM (green dotted line), and $\Gamma R$ (red dash-dot line). Theoretical bandgaps are outlined in gray.

Measurements are reported in Fig. 5(a). The harmonic velocity $\omega\left|u_{z}\right|$ is plotted as a function of frequency. Vibrations at the bottom surface were measured to check the frequency response of the PZT transducer. The wide bandwidth emission of elastic waves covers from almost a few $\mathrm{KHz}$ up to $10 \mathrm{MHz}$. The frequency response of the phononic crystal is compatible with a wide bandgap extending approximately from $0.6 \mathrm{MHz}$ to $7.5 \mathrm{MHz}$. Significantly, there is a sharp drop at the bandgap entrance and a transmission recovery above the bandgap, peaking around 8.5 MHz. The numerical displacement transmission plotted in Fig. 5(b) confirms those observations.

In this letter, we have outlined the design of an ultrawide bandgap 3D phononic crystal for ultrasonic waves in the $\mathrm{MHz}$ range. Samples with complex unit cells were manufactured using two-photon lithography with lattice constants in the range of hundreds of micrometers. The experimental characterisation unambiguously shows a bandgap extending from $0.6 \mathrm{MHz}$ to $7.5 \mathrm{MHz}$. This study paves the way for the use of three dimensional 
two-photon lithography for ultrasonic applications.

\section{ACKNOWLEDGEMENTS}

We thank Franck Lardet-Vieudrin for help with the MEMS analyzer operation. We acknowledge support by the EIPHI Graduate School (contract ANR-17-EURE0002) and the French Investissements d'Avenir program, project ISITE-BFC (contract ANR-15-IDEX-03). This work was supported by the french RENATECH network and its FEMTO-ST technological facility.

\section{DATA AVAILABILITY STATEMENT}

The data that support the findings of this study are available from the corresponding author upon reasonable request.

\section{REFERENCES}

${ }^{1}$ M. S. Kushwaha, P. Halevi, L. Dobrzynski, and B. Djafari-Rouhani, "Acoustic band structure of periodic elastic composites," Phys. Rev. Lett. 71, 20222025 (1993).

${ }^{2}$ V. Laude, Phononic crystals: artificial crystals for sonic, acoustic, and elastic waves, 2nd Ed. (Walter de Gruyter, Berlin, 2020).

${ }^{3}$ G. W. Milton, The Theory of Composites (Cambridge University Press, 2002).

${ }^{4} \mathrm{~J}$. Li and C. T. Chan, "Double-negative acoustic metamaterial," Phys. Rev. E 70, 055602 (2004).

${ }^{5}$ W. Cai and V. M. Shalaev, Optical metamaterials, Vol. 10 (Springer, 2010).

${ }^{6} \mathrm{C}$. Soukoulis and M. Wegener, "Past achievements and future challenges in the development of threedimensional photonic metamaterials," Nat. Photonics 5, 523 (2011).

${ }^{7}$ M. Kadic, R. Schittny, T. Bückmann, C. Kern, and M. Wegener, "Hall-effect sign inversion in a realizable 3D metamaterial," Physical Review X 5, 021030 (2015).

${ }^{8}$ K. Bertoldi, V. Vitelli, J. Christensen, and M. van Hecke, "Flexible mechanical metamaterials," Nat. Rev. Mater. 2, 1-11 (2017).

${ }^{9}$ J. Christensen, M. Kadic, O. Kraft, and M. Wegener, "Vibrant times for mechanical metamaterials," MRS Commun. 5, 453-462 (2015).

${ }^{10}$ X. Chen, Q. Ji, J. Wei, H. Tan, J. Yu, P. Zhang, V. Laude, and M. Kadic, "Light-weight shell-lattice metamaterials for mechanical shock absorption," International Journal of Mechanical Sciences 169, 105288 (2020).

${ }^{11}$ M. Kadic, G. Milton, M. van Hecke, and M. Wegener, "3D metamaterials," Nat. Rev. Phys. 1, 198-210 (2019).
${ }^{12}$ R. Süsstrunk and S. D. Huber, "Classification of topological phonons in linear mechanical metamaterials," Proceedings of the National Academy of Sciences 113, E4767-E4775 (2016).

${ }^{13}$ T. Frenzel, M. Kadic, and M. Wegener, "Threedimensional mechanical metamaterials with a twist," Science 358, 1072-1074 (2017).

${ }^{14} \mathrm{~T}$. Frenzel, J. Köpfler, E. Jung, M. Kadic, and M. Wegener, "Ultrasound experiments on acoustical activity in chiral mechanical metamaterials," Nat. Commun. 10, 3384 (2019).

${ }^{15}$ Y. Chen, T. Frenzel, S. Guenneau, M. Kadic, and M. Wegener, "Mapping acoustical activity in 3D chiral mechanical metamaterials onto micropolar continuum elasticity," J. Mech. Phys. Solids 137, 103877 (2020).

${ }^{16}$ Y. Chen, M. Kadic, S. Guenneau, and M. Wegener, "Isotropic chiral acoustic phonons in 3D quasicrystalline metamaterials," Phys. Rev. Lett. 124, 235502 (2020).

${ }^{17}$ Z. Liu, X. Zhang, Y. Mao, Y. Y. Zhu, Z. Yang, C. T. Chan, and P. Sheng, "Locally resonant sonic materials," Science 289, 1734-1736 (2000).

${ }^{18}$ R. V. Craster and S. Guenneau, Acoustic Metamaterials, Springer series in materials science (Springer, 2013).

${ }^{19}$ T. L. Szabo, Diagnostic ultrasound imaging: inside out (Academic Press, 2004).

${ }^{20}$ K. K. Shung, Diagnostic ultrasound: Imaging and blood flow measurements (CRC press, 2015).

${ }^{21}$ A. Khelif, F.-L. Hsiao, A. Choujaa, S. Benchabane, and S. Laude, "Octave omnidirectional band gap in a threedimensional phononic crystal," IEEE Trans. Ultrason., Ferroelec., Freq. Control 57, 1621 (2010).

${ }^{22}$ I. K. Tragazikis, D. A. Exarchos, P. T. Dalla, K. Dassios, T. E. Matikas, and I. E. Psarobas, "Elastodynamic response of three-dimensional phononic crystals using laser Doppler vibrometry," J. Phys. D: Appl. Phys. 52, 285305 (2019).

${ }^{23}$ L. D'Alessandro, E. Belloni, R. Ardito, A. Corigliano, and F. Braghin, "Modeling and experimental verification of an ultra-wide bandgap in 3D phononic crystal," Appl. Phys. Lett. 109, 221907 (2016).

${ }^{24}$ F. Warmuth, M. Wormser, and C. Körner, "Single phase 3D phononic band gap material," Scientific Reports 7, 3843 (2017).

${ }^{25}$ F. Lucklum and M. J. Vellekoop, "Bandgap engineering of three-dimensional phononic crystals in a simple cubic lattice," Appl. Phys. Lett. 113, 201902 (2018).

${ }^{26}$ O. McGee, H. Jiang, F. Qian, Z. Jia, L. Wang, H. Meng, D. Chronopoulos, Y. Chen, and L. Zuo, "3D printed architected hollow sphere foams with lowfrequency phononic band gaps," Additive Manufacturing 30, 100842 (2019).

${ }^{27}$ L. Yang, A. Münchinger, M. Kadic, V. Hahn, F. Mayer, E. Blasco, C. Barner-Kowollik, and M. Wegener, "On the schwarzschild effect in 3D two-photon laser lithography," Advanced Optical Materials 7, 1901040 (2019). 
${ }^{28}$ P. Hagedorn and A. DasGupta, Vibrations and waves in continuous mechanical systems (Wiley Online Library, 2007) p. 127.

${ }^{29}$ S. I. Ranganathan and M. Ostoja-Starzewski, "Universal elastic anisotropy index," Phys. Rev. Lett. 101,
055504 (2008).

${ }^{30}$ Z. Jia, Y. Chen, H. Yang, and L. Wang, "Designing phononic crystals with wide and robust band gaps," Phys. Rev. Applied 9, 044021 (2018). 